\title{
DIVISION X WORKING GROUP on HISTORIC RADIO ASTRONOMY
}

\author{
CHAIR \\ VICE-CHAIR \\ MEMBERS
}

\author{
Wayne Orchiston \\ Kenneth I. Kellermann \\ Rodney D. Davies, Suzanne V. Débarbat, \\ Masaki Morimoto, Slava Slysh, \\ Govind Swarup, Hugo van Woerden, \\ Jasper V. Wall, Richard Wielebinski
}

PROCEEDINGS BUSINESS SESSIONS, August 2009

\section{Introduction}

During the Rio General Assembly we held the following meetings of the Working Group: a Business Meeting, a Science Meeting on "The Development of Aperture Synthesis Imaging in Radio Astronomy", and a Science Meeting on "Recent Research".

\section{Business Meeting}

This meeting was held on Tuesday 4 August, during Session 2. The contents of the WG's Report included in the Division's Triennial Report were summarised. Despite representations from the WG, Stanford University decided to demolish five 60-ft antennas at the Highway 280 site, although the concrete piers with carved names of noted optical and radio astronomers did survive. During the Triennium research on the history of radio astronomy was carried out by more than 40 different astronomers from nine countries, and various papers were published in the Journal of Astronomical History and Heritage. Papers from the Science Meeting on European Radio Astronomy held at the Prague General Assembly were published in a special addition of Astronomische Nachrichten (edited by Richard Wielebinski, Ken Kellermann and Wayne Orchiston). Deaths of the following colleagues during the Triennium were reported: Ron Bracewell, Chris Christiansen, Fred Haddock, Valadimir Kotelnikov, John Kraus, Arkady Kuzmin, Lex Muller, Brian Robinson, Slava Slysh, Henk van der Hulst and Paul Wild.

The Program of Work for the WG during the 2009-2012 Triennium was outlined and discussed, based on the successful program of 2006-2009. A number of new research initiatives were announced.

The following WG Committee for the 2009-2012 Triennium was announced:

Ken Kellermann (Chair - USA), Wayne Orchiston (Vice-Chair - Australia), Rod Davies (UK), James Lequeux (France), Norio Kaifu (Japan), Yuri Ilyasov (Russia), Govind Swarup (India), Hugo van Woerden (The Netherlands), Jasper Wall (Canada) and Richard Wielebinski (Germany)

Under General Business the release of a history of radio astronomy DVD and forthcoming books was announced, and Ken Kellermann reported that NRAO is digitizing early radio astronomy courses from Westfold (Caltech), van der Hulst (Harvard) and Barrett (MIT). 


\section{Science meeting on "The development of synthesis imaging in radio astronomy"}

This meeting was held on Wednesday 5 August during Sessions 3 and 4, and included the presentation of the 2009 Grote Reber Medal to Barry Clark. Six papers (by Barry Clark, Tim Cornwall, Ron Ekers, Bob Frater, Miller Goss, and Radhakristian) were presented during this meeting.

\section{Science meeting on "Recent research"}

This meeting was also held on Wednesday 5 August, during Session 2. Four papers (by John Dickel, Bruce McAdam, Harry Wendt, Wayne Orchiston, Bruce Slee, Ron Stewart and Harry Wendt) were presented, and four poster papers (by Martin George, Peter Robertson, Wayne Orchiston, Bruce Slee, Ron Stewart and Harry Wendt) were displayed.

Wayne Orchiston Chairman of the Working Group

Ken Kellermann Vice-Chairman of the Working Group 